\title{
Finally, a Minimally Invasive Option for Intrahepatic Inferior Vena Cava Invasion by Hepatocellular Carcinoma
}

\author{
Erik Soule Jerry Matteo \\ Department of Interventional Radiology, UF Health Jacksonville, University of Florida, \\ Jacksonville, FL, USA
}

\section{Keywords}

Hepatocellular carcinoma - Cryoablation - Intrahepatic inferior vena cava

\begin{abstract}
Background: Major vessel invasion is a late manifestation of hepatocellular carcinoma, which may directly result in mortality if left untreated. Surgical resection may be an option for select patients; however, surgery may be contraindicated. Contraindications include multifocal disease, poor liver reserve, inability to tolerate surgery, and patient preference. Cryoablation is a minimally invasive therapy utilized for treating hepatic neoplasms by subjecting them to extreme cold temperatures. The "thermal sink" effect describes cryoablation near flowing blood such as that found in high volumes within major vascular structures. Thermodynamics dictates that proximity to the flowing intravascular compartment will decrease ice formation, and therefore, tumor destruction. Methods: This report describes a novel palliative technique to induce temporary cessation of the blood flow in the intrahepatic inferior vena cava (IVC), thus enabling the operator to perform cryoablation targeting invasive cancer within. Results: Adequate ablation using this technique allowed a patient with IVC invasion 9 months of survival. Conclusion: Quality of life during this time was maximized, as recovery time from this procedure is minimal.

(C) 2018 S. Karger AG, Base
\end{abstract}

\section{Introduction}

Hepatocellular carcinoma (HCC) is a dreaded complication of liver cirrhosis, which accounts for approximately 800,000 deaths annually worldwide [1]. The median survival after diagnosis ranges from 6 to 20 months. The high case fatality rate reflects the relentless 
nature of the disease, which typically invades locally and frequently recurs after treatment. Macrovascular invasion indicates an aggressive disease phenotype and is seen in as many as $35 \%$ of the patients [2]. Inferior vena cava (IVC) invasion portends a very poor prognosis, with a mean survival time $<3$ months [3]. Resection is increasingly being utilized for patients with IVC metastasis by experienced surgeons in high-incidence regions, but this remains a contraindication to surgery at many institutions. Multifocal disease and poor hepatic reserve due to cirrhosis or large tumor burden remain as barriers to surgical intervention. Additionally, quality of life concerns for patients unlikely to achieve curative resection may factor into a risk/benefit stratification. Resection of great vessel HCC metastasis is a high-risk surgery; many patients will not be acceptable candidates. Some may elect for a palliative approach; however, HCC is notoriously unresponsive to systemic therapies. This article details a novel palliative technique, which may prolong survival in patients with intrahepatic IVC invasion while preserving their quality of life.

Percutaneous cryoablation (PC) is a minimally invasive modality increasingly utilized for the treatment of primary and metastatic neoplastic lesions. Advantages of PC include intrinsic analgesic properties [4], intraoperative imaging surveillance of the ablation zone [5], potential for tumor-antigen-specific immune response [6], versatility in targeting surgically inaccessible malignancy [7], and tolerability for poor surgical candidates [8]. PC achieves cytoreduction of the tumor burden through a combination of membrane rupture due to crystal formation at the center of the ice ball and apoptosis at the periphery where freeze rates are insufficient to disrupt membrane integrity. Post-thaw ischemia also contributes to cell death: microvasculature up to $180 \mu \mathrm{m}$ in diameter is obliterated by PC [9]. Larger vessels within the ablation zone are initially patent, although likely irreparably damaged. Two weeks after cryoablation, a flow void is seen on intravenous contrast-enhanced imaging studies [10]. This may be due to freezing-induced endothelial damage and thrombosis, occurring even in the 5-to10-mm periphery of the ice ball, which fails to reach cytotoxic temperatures [11].

Major vascular structures, such as the aorta, superior vena cava (SVC), and IVC, however, appear to be protected by heat dissipation due to brisk flow. This is known as the "thermal sink" effect. Histologic analyses following cryoablative insult have shown initial endothelial and smooth muscle cell death with maintenance of vessel patency through preserved elastin fibers. A single layer of platelets binds to these exposed fibers, which are replaced after about 1 week by migrating endothelial cells from adjacent areas. Smooth muscle regeneration occurs after about 2 weeks through subendothelial migration and subsequent proliferation, and complete repair is evident after around 1 month [12]. The "thermal sink" effect has been postulated to account for local cancer recurrence due to inadequate treatment adjacent to major vascular structures $[13,14]$. Emerging techniques have been described in the literature, but until now, none have been able to confidently conquer this problem. We describe a technique using a large angioplasty balloon causing temporary cessation of the blood flow to allow intravascular cyroablation of a tumor extension within the intrahepatic IVC.

\section{Materials and Methods}

The patient was a 56-year-old female with hepatitis $\mathrm{C}$ and liver cirrhosis who initially presented with a 7-cm HCC in segment 4, as well as several smaller satellite lesions. The tumor was deemed unresectable, she was not a transplant candidate, and she refused systemic therapy. Life expectancy was initially determined to be $<3$ months at multidisciplinary tumor board meetings. She was referred to interventional radiology and underwent 3 transarterial chemoembolizations, followed by 3 hepatic cryoablations over the course of several years with adequate ablation. Recurrent disease was subsequently identified on surveillance imaging and found to be invading the intrahepatic IVC (Fig. 1a-c). Moderate sedation was utilized in the performance of this procedure. Pre- and postsedation patient evaluation, administration of the sedative and analgesic 
Gastro

Intestinal

Tumors

Fig. 1. a Coronal CT image showing HCC extension into the IVC without tumor thrombus. b, c Axial CT images demonstrating HCC extension into the IVC without tumor thrombus.

\begin{tabular}{l|l}
\hline Gastrointest Tumors 2018;5:54-61 \\
\hline DOI: 10.1159/000491694 & $\begin{array}{l}\text { @ 2018 S. Karger AG, Basel } \\
\text { www.karger.com/gat }\end{array}$ \\
\hline
\end{tabular}

Soule and Matteo: Finally, a Minimally Invasive Option for Intrahepatic Inferior Vena Cava Invasion by Hepatocellular Carcinoma
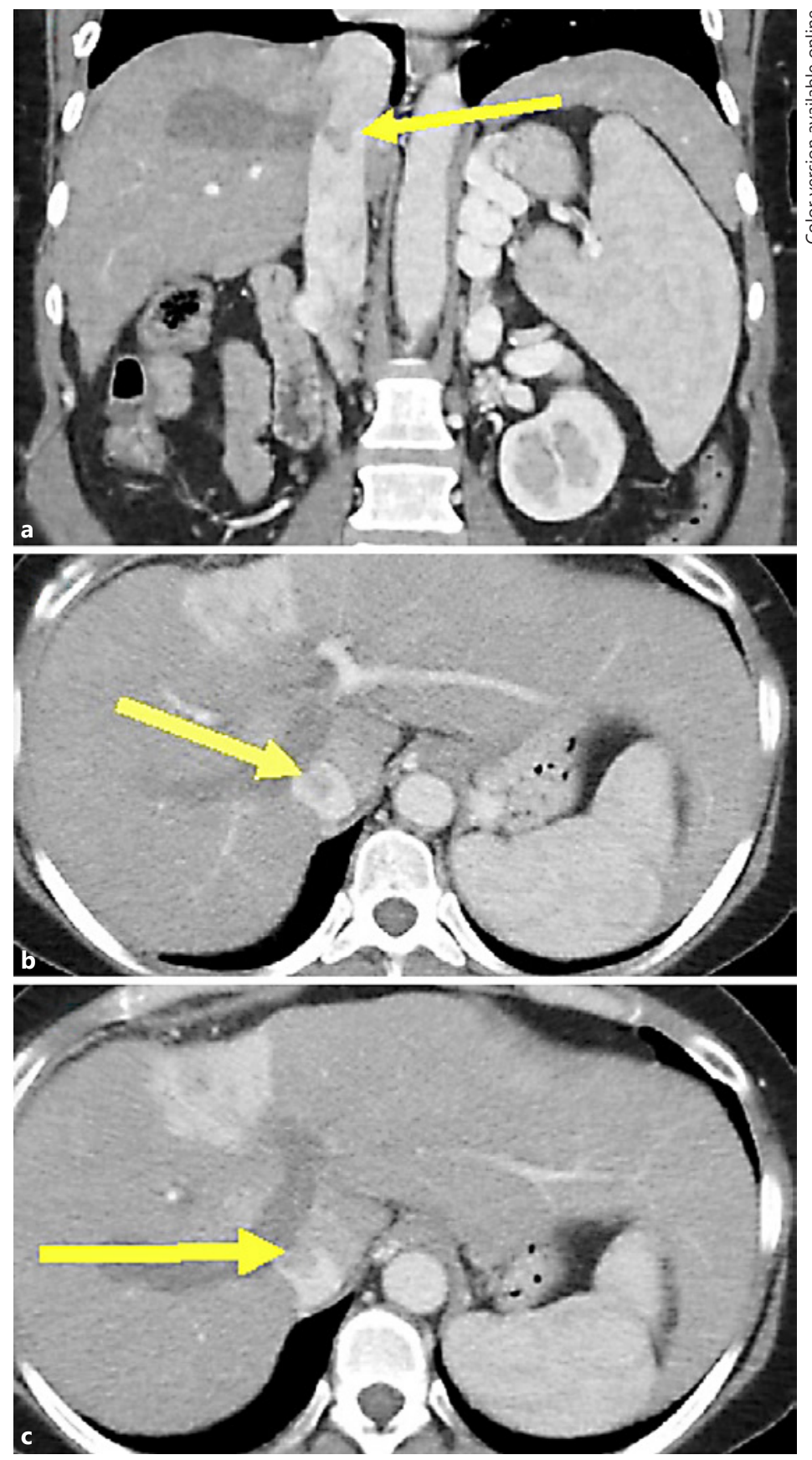

agents, and monitoring of the cardiorespiratory function were performed with the assistance of an independent, trained observer.

After appropriate informed consent was obtained, the patient was placed supine on the angiography table. The right groin was prepared and draped in a sterile fashion. The skin overlying the common femoral vein was then anesthetized with lidocaine. Access was obtained to the common femoral vein using a singlewall technique, and a guidewire was introduced into the IVC. Over the guidewire, a Q50 balloon catheter (W.L. Gore, Flagstaff, AZ, USA) was introduced into the IVC between the renal vein confluence and the intrahepatic segment, and a venogram was performed via the balloon catheter. This demonstrated a filling defect consistent with intraluminal tumor within the intrahepatic segment of the IVC; no filling defects were identified in the infra-/suprahepatic IVC (Fig. 2a). 


\section{Gastro Intestinal \\ Tumors}
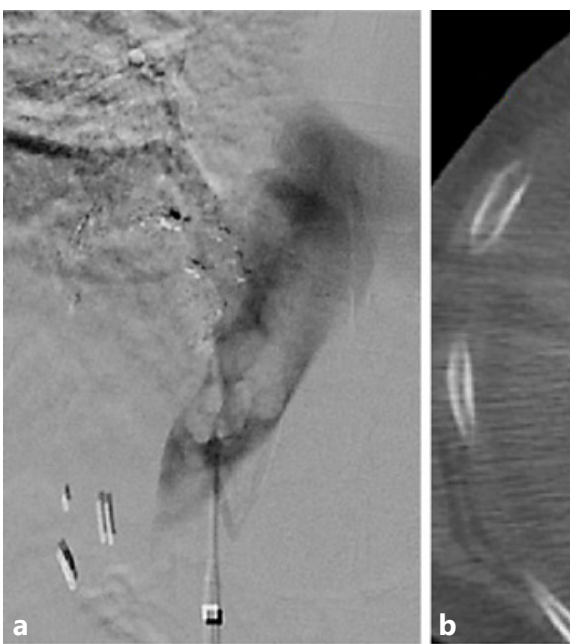

\begin{tabular}{|c|c|}
\hline DOI: 10.1159/000491694 & $\begin{array}{l}\text { (c) } 2018 \text { S. Karger AG, Basel } \\
\text { www.karger.com/gat }\end{array}$ \\
\hline
\end{tabular}

Soule and Matteo: Finally, a Minimally Invasive Option for Intrahepatic Inferior Vena Cava Invasion by Hepatocellular Carcinoma
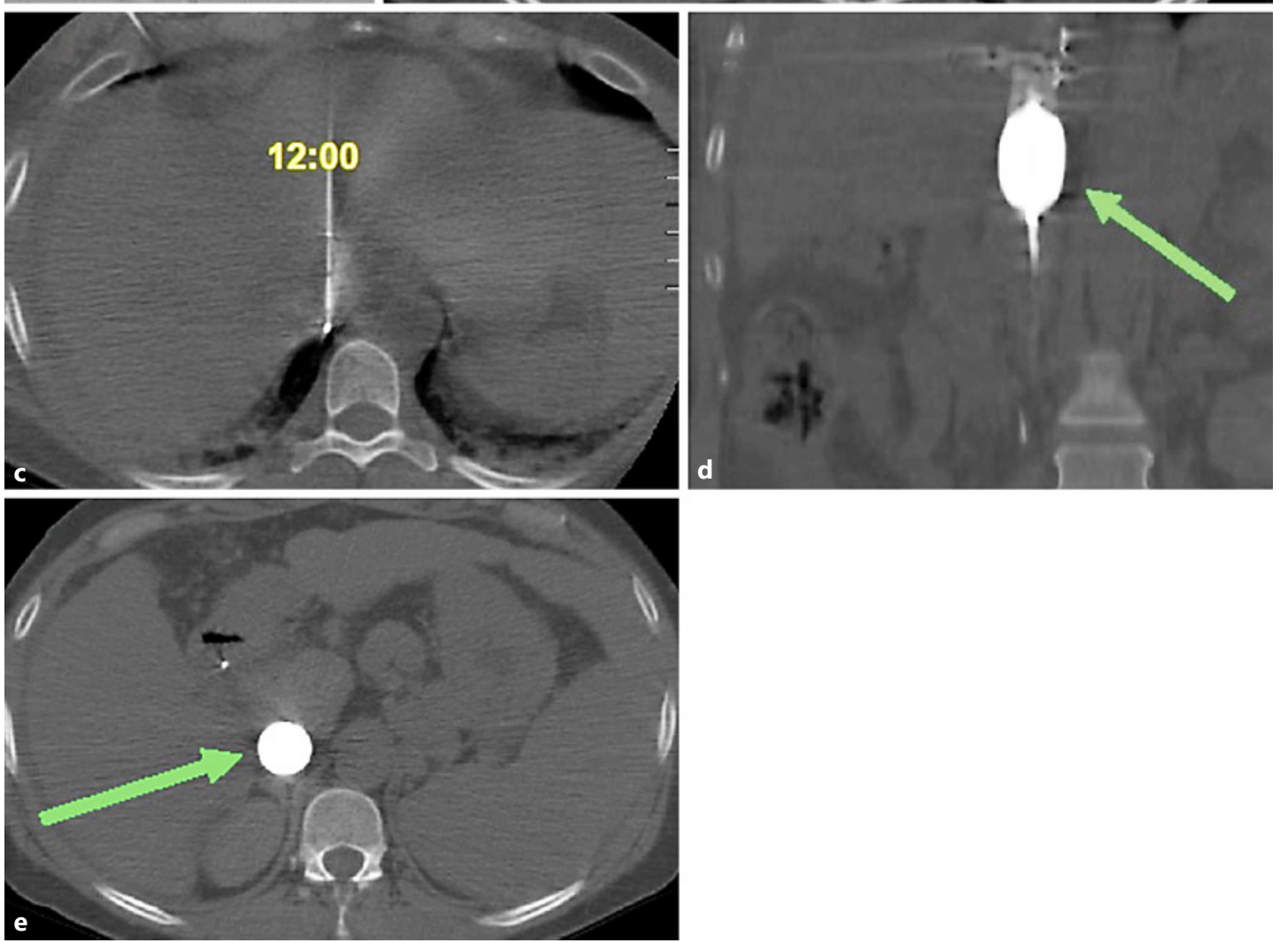

Fig. 2. a IVC venogram showing HCC extension into the IVC without tumor thrombus. b, c Intraoperative axial CT scan demonstrating 3 cryoprobes placed at the 10:00,11:00, and 12:00 positions to completely ablate the tumor. d, e Coronal, axial CT showing a Q50 balloon inflated within the suprarenal IVC for intermittent cessation of the blood flow during cryoablation.

The patient was then transported to the computed tomography (CT) scanner, positioned supine on the CT gantry, and the right upper quadrant abdomen was prepared and draped in the usual sterile fashion. Limited CT images were performed to delineate the liver mass and for procedure planning. Additional images were obtained following the administration of intravenous contrast via the previously placed catheter for delineation of the intravascular tumor. The skin was marked. Using CT guidance, three 17-gauge cryoablation probes were inserted using direct CT fluoroscopy into the liver mass bracketing the tumor, one in the superior 
portion of the tumor including the intravascular component, the second within the mid portion of the tumor, and the third within the inferior portion of the tumor (Fig. 2b, c).

The Q50 balloon catheter was inflated during the freezing portion of the procedure to the limit blood flow and to decrease thawing of the ice ball (Fig. 2d, e). Three freeze cycles were performed with durations of 3 min each. The freeze cycles were interspersed with 30-s thaw cycles during which the balloon was deflated. Limited CT images were obtained at the 5-min interval to evaluate the progression of the ice ball. This demonstrated expected coverage of the liver mass. Following removal of the cryoablation probes, limited CT was performed revealing hypodensity within the region of the liver mass consistent with the ice ball. No complications or injury to surrounding organs were noted. The cryoablation probes, balloon catheter, and the right groin sheath were removed, and sterile dressings were applied.

\section{Results}

The patient tolerated the procedure well without immediate complications. Following completion of the procedure, the patient was admitted for overnight observation and pain control, and was discharged the next day. Follow-up imaging at 7 months after the procedure demonstrated continued decrease in the size of the tumor and no treatment-related complications (Fig. 3a-c). The patient survived 9 months after the procedure with a good quality of life. She was able to travel with her family and retained good functional status until eventually succumbing to liver failure from her aggressive, multifocal HCC.

\section{Discussion}

HCC may invade major vessels through stromal destruction, with endothelial rupture and formation of tumor thrombus. Alternatively, clusters of tumor cells may be found in the vascular lumen, which are coated with endothelium and thus are protected from initiation of the coagulation cascade. This may occur in the absence of stromal destruction near the tumor periphery and is hypothesized to enhance the propensity for distant metastasis [15]. Indeed, microemboli encapsulated in endothelium were observed herniating into peritumoral vessels and were subsequently able to be isolated from the blood. They may retain viability and metastatic potential upon deposition in the pulmonary and hepatic capillary beds, thus demonstrating a metastatic mechanism independent from epithelial-mesenchymal transition [16]. The detection of endothelium-coated microemboli in adjacent nontumor tissue served as an independent predictor of poor prognosis after resection. Overall survival and the time to recurrence were decreased, in contrast to cases in which uncoated microemboli were observed [17].

Enhanced metastatic potential of HCC clones that herniate into vessels expressing an endothelial coating can be attributed in part to cloaking of their antigens from the immunosurveillance mechanism. The endothelial coating itself may also contribute, as it provides protection from shear stress, complement proteins, and clotting factors encountered in the circulation. HCC cells secreting an endothelial vascular invasion membrane may skip a number of steps in the cancer evolution pathway, which they would otherwise need to survive independently for long enough to establish bloodborne metastases. This process may be facilitated by HCC expression of angiopoetin-2, which plays a role in the activation of the vascular endothelial growth factor (VEGF) pathway. Angiopoetin-2 expression may enable HCC to package clusters of malignant cells into raft-like endothelial structures and push them into peritumoral vessels [16]. Increased intratumoral pressure from abnormal growth within the neoplasm may facilitate this process, along with other characteristics of the tumor microenvironment such as tissue hypoxia [18]. 
Gastro

Intestinal

Tumors

Fig. 3. a Follow-up axial CT image 6 weeks after cryoablation. b Follow-up axial CT image 12 weeks after cryoablation. c Follow-up axial CT image 7 months after cryoablation.

\begin{tabular}{l|l}
\hline Gastrointest Tumors 2018;5:54-61 \\
\hline DOI: 10.1159/000491694 & $\begin{array}{l}\text { @ 2018 S. Karger AG, Basel } \\
\text { www.karger.com/gat }\end{array}$ \\
\hline
\end{tabular}

Soule and Matteo: Finally, a Minimally Invasive Option for Intrahepatic Inferior Vena Cava Invasion by Hepatocellular Carcinoma
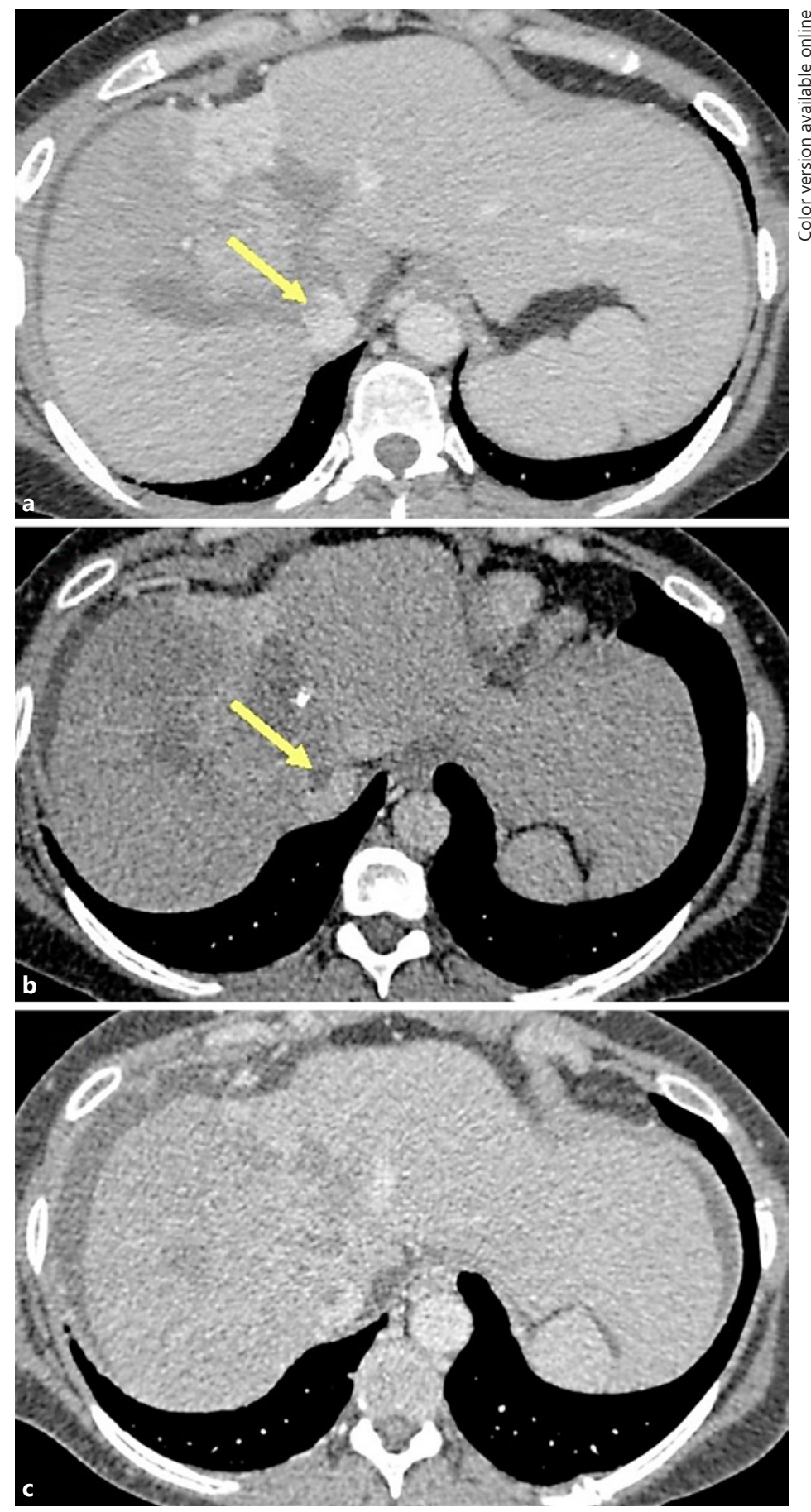

Hypoxia drives angiogenesis, characterized by abnormal vascular remodeling and 'leaky' tumor vasculature. This manifests as local hypoxia in underperfused portions of the tumor, in turn upregulating the expression of VEGF. Elevated VEGF expression correlates with propensity for vascular invasion, recurrence after treatment, and is a predictor of poor prognosis. Indeed, VEGF overexpression in cirrhotic livers has been implicated in hepatic carcinogenesis [19]. The positive feedback loop described involving angiogenesis and hypoxia has disastrous implications for patients and is a major contributor to HCC's dreadful prognosis. 
Sorafenib, purported as a VEGF pathway inhibitor is being deployed in clinical practice for patients with advanced HCC and has been shown to increase overall survival. Interventional oncologic techniques, such as transarterial chemoembolizations, radioembolization, and cryoablation in combination with sorafenib, to transiently normalize tumor vasculature may be employed to treat advanced HCC. Even in the setting of intrahepatic IVC invasion, intermittent flow obstruction using an angioplasty balloon may facilitate palliative cryoablation.

\section{Conclusion}

Intravascular cryoablation is possible utilizing an intermittently inflated angioplasty balloon within the IVC. This method provides a technical strategy to overcome the 'thermal sink' phenomenon by causing temporary cessation of the blood flow. Patients with intrahepatic IVC invasion may benefit from this modality, if their neoplasm is not amenable to other ablative, embolization, or surgical methods.

\section{Statement of Ethics}

Informed, written consent was obtained from the patient prior to participation in this study. This case was reviewed by UF IRB-03 Chairman and it was determined that as a single-case report, it does not require IRB approval.

\section{Disclosure Statement}

The authors declare that they have no conflicts of interest to disclose.

\section{References}

1 Fitzmaurice C, et al: The burden of primary liver cancer and underlying etiologies from 1990 to 2015 at the global, regional, and national level: results from the Global Burden of Disease Study 2015. JAMA Oncology 2017;3:1683-1691.

2 Lee $\mathrm{Y}$, et al: Vascular invasion in hepatocellular carcinoma prevalence, determinants, and prognostic impact. J Clin Gastroenterol 2014;48:734-741.

3 Jun C, et al: Risk factors for patients with stage IVB hepatocellular carcinoma and extension into the heart: prognostic and therapeutic implications. Yonsei Med J 2014;55:379-386.

4 Thacker PG, et al: Palliation of painful metastatic disease involving bone with imaging-guided treatment: comparison of patients' immediate response to radiofrequency ablation and cryoablation. AJR Am J Roentgenol 2011;197:510-515

5 Callstrom M, Kurup A: Percutaneous ablation for bone and soft tissue metastases - why cryoablation? Skeletal Radiol 2009;38:835-839.

6 Sabel MS: Cryo-immunology: a review of the literature and proposed mechanisms for stimulatory versus suppressive immune responses. Cryobiology 2009;58:1-11.

7 Soule E, et al: Freezing Fort Knox - mesenteric carcinoid cryoablation. Gastrointest Tumors 2017;4:53-60.

8 Littrup PJ, et al: Soft-tissue cryoablation in diffuse locations: feasibility and intermediate term outcomes. J Vasc Interv Radiol 2013;24:1817-1825.

9 Lagerveld BW, et al: Immediate effect of kidney cryoablation on renal arterial structure in a porcine model studied by imaging cryomicrotome. J Urol 2010;183:1221-1226.

10 Lagerveld BW, et al: Gradient changes in porcine renal arterial vascular anatomy and blood flow after cryoablation. J Urol 2011;186:681-686.

11 Sainani NI, et al: Incidence and clinical sequelae of portal and hepatic venous thrombosis following percutaneous cryoablation of liver tumors. Abdom Radiol 2016;41:970-977.

12 Ladd AP, et al: Cryosurgical effects on growing vessels. Am Surg 1999;65:677-682.

13 Colak E, et al: CT-guided percutaneous cryoablation of central lung tumors. Diagn Interv Radiol 2014;20: 316-322. 
14 Garnon J, et al: Percutaneous image-guided cryoablation of challenging mediastinal lesions using large-volume hydrodissection: technical considerations and outcomes. Cardiovasc Intervent Radiol 2016;39:1636-1643.

15 Quaglia A, et al: Vascular invasion and herniation by hepatocellular carcinoma in cirrhosis: a wolf in sheep's clothing. Arch Pathol Lab Med 2005;129:639-644.

16 Fang JH, et al: A novel vascular pattern promotes metastasis of hepatocellular carcinoma in an epithelialmesenchymal transition-independent manner. Hepatology 2015;62:452-465.

17 Ding T, et al: Endothelium-coated tumor clusters are associated with poor prognosis and micrometastasis of hepatocellular carcinoma after resection. Cancer 2011;117:4878-4889.

18 Ivey JW, et al: Improving cancer therapies by targeting the physical and chemical hallmarks of the tumor microenvironment. Cancer Lett 2016;380:330-339.

19 Zhu AX, et al: HCC and angiogenesis: possible targets and future directions. Nat Rev Clin Oncol 2011;8:292301. 